\title{
LES ÉCHECS DES TENTATIVES DE RÉGULATION INTERNATIONALE
}

\section{Daniel Cornu $^{1}$}

Au cours du $\mathrm{XX}^{\mathrm{e}}$ siècle, plusieurs initiatives ont été prises au niveau international, en vue de formuler des règles de conduite communes et de mettre en place des instances de régulation du journalisme. Les plus significatives furent le projet d'un code d'honneur international du personnel de presse et d'information, développé au début des années cinquante sous l'autorité de l'Organisation des Nations Unies ; la définition en 1983 de principes internationaux de l'éthique professionnelle des journalistes, fondés sur la Déclaration de l'UNESCO de 1978 sur le «Nouvel ordre mondial de l'information et de la communication»; la résolution du Conseil de l'Europe de 1994 demandant la définition d'un code de déontologie applicable aux pays membres. Ces tentatives d'installer une régulation par le haut ont toutes échoué. Elles restent cependant intéressantes par ce qu'elles révèlent des attentes concernant les médias. Et plus encore par ce que confirme leur échec même: l'attachement à une conception libérale, qui fonde toute forme de régulation autre que le respect des lois sur une discipline interne et librement consentie.

1 Directeur du Centre romand de formation des journalistes, professeur à l'Université de Neuchâtel.

Recherches en communication, $\mathrm{n}^{\circ} 9,(1998)$. 


\section{L'action de l'UNESCO}

Le projet des Nations Unies aboutit en 1952 à la rédaction d'un texte qui ne fut jamais appliqué ; il concernait à la fois les journalistes et les fonctionnaires employés à la communication officielle, statut courant dans les pays communistes. Il était normal dès lors que le traitement des questions touchant à l'information et à la communication revînt à l'UNESCO. L'action de cette organisation a dominé la discussion sur l'éthique de l'information dans les années soixante-dix et quatre-vingt. Elle se déroula en trois phases.

$\mathrm{Au}$ cours de la première phase, l'UNESCO entreprit de définir une plate-forme déontologique commune aux deux principales organisations internationales de journalistes. La «Déclaration de Bordeaux» de la Fédération internationale des journalistes (F.I.J.), installée à Bruxelles, s'inscrivait dans une ligne libérale. Elle précisait que «le journaliste n'accepte, en matière professionnelle, que la juridiction de ses pairs, à l'exclusion de toute intrusion gouvernementale ou autre». A l'inverse, les positions prises par l'Organisation internationale des journalistes (O.I.J.), dont le siège était à Prague, soumettaient l'éthique journalistique à des visées étrangères au strict accomplissement des devoirs professionnels. Elles cherchaient à définir les termes de la responsabilité journalistique en accord avec des objectifs politiques : aspiration des peuples à l'indépendance nationale, progrès social, démocratie, liberté et paix. Comment concilier ces points de vue?

Une réunion consultative eut lieu à la Maison de l'UNESCO, à Paris, les 12 et 13 novembre 1973. Elle réunissait les représentants d'organisations internationales non gouvernementales de presse, de radio et de télévision. Les participants examinèrent les codes adoptés par les organisations professionnelles dans les diverses régions du monde. Plusieurs solutions furent envisagées afin d'harmoniser les formulations de la déontologie : un code unique, qui pourrait servir de modèle à tous; un code dont certains articles seraient modulés afin de répondre aux besoins variables d'organisations appartenant à des pays et des systèmes économiques et sociaux différents; une liste de principes très généraux qui pourraient être adoptés, totalement ou en partie seulement, par les organisations de la presse, de la radio, de la télévision, ainsi que du cinéma et de la publicité.

Le résultat fut une «mise à plat» des principes déontologiques, ainsi qu'un accord sur les règles principales devant orienter les 
pratiques des médias. L'apport le plus positif fut un essai de classification des devoirs professionnels, selon qu'ils incombent aux agents des médias ou aux responsables de la gestion des entreprises de presse, soit aux médias comme organisations. L'effet concret se limita à la rédaction d'un document conclusif.

Les questions posées par les approches concurrentes des organisations professionnelles de l'Est et de l'Ouest restaient donc ouvertes. C'est sur ce terrain que se sont déroulées, lors d'une deuxième phase, les discussions sur le «Nouvel ordre mondial de l'information et de la communication» ou «NOMIC».

L'Union soviétique et ses alliés entreprirent dans les années soixante-dix une vaste manœuvre visant à légitimer le contrôle étatique des médias et à renforcer leur monopole dans le domaine de l'information du public. Il s'agissait en somme d'opérer un renversement de la tendance libérale jusqu'ici suivie par les institutions du système des Nations Unies, qui laissait aux professionnels eux-mêmes la responsabilité de définir leur pratique et d'en établir les règles.

L'habileté politique de l'Union soviétique fut de prendre appui sur la situation de l'hémisphère Sud. L'argument était en soi peu contestable et reste largement pertinent. Il existe entre les pays industrialisés et les pays en voie de développement une différence de moyens financiers, techniques et humains qui entraîne une distorsion énorme dans le flux des informations. Dans les années soixante-dix, seuls quelques grands pays occidentaux, en particulier les États-Unis, la Grande-Bretagne et la France, ainsi que l'Union soviétique ellemême, étaient en mesure de constituer de véritables réseaux d'information planétaires. Bien qu'ils fussent édifiés sur les principes du libéralisme et du pluralisme, les réseaux occidentaux jouissaient de fait d'une position dominante dans le monde. Ils ne renseignaient pas seulement les opinions publiques de leurs pays respectifs ; ils géraient le marché de l'information dans le monde, et donc aussi dans le tiers monde. Ils produisaient alors un effet double : non seulement le tiers monde n'avait pas les moyens de donner au reste du «village planétaire» sa propre version des faits, sa propre vision de l'actualité, dans un esprit pluraliste, mais encore les moyens lui manquaient de se renseigner lui-même sur ses propres événements.

En usant de l'argument qu'il ne pouvait y avoir de libre circulation de l'information aussi longtemps que sa diffusion serait à ce point déséquilibrée au profit des pays du Nord, l'Union soviétique visait en fait à limiter, et si possible à empêcher, la pénétration dans sa propre 
sphère d'influence des grands médias occidentaux, en particulier des agences internationales.

L'offensive partit de loin, puisqu'une résolution adoptée par l'UNESCO, lors de la seizième session de la Conférence générale de 1970, invitait déjà les États membres «à prendre les dispositions nécessaires, notamment d'ordre législatif, en vue d'encourager l'emploi des moyens d'information contre la propagande en faveur de la guerre, du racisme et de la haine entre nations». Lors de la session suivante, en 1972, une autre résolution demanda la préparation d'un projet de déclaration «contenant les principes fondamentaux de l'emploi des moyens de grande information en vue du renforcement de la paix, de la compréhension internationale et de la lutte contre la propagande belliciste, le racisme et l'apartheid».

L'entreprise prit une tournure concrète lors de la dix-neuvième session de la Conférence générale, en 1976 à Nairobi, au cours de laquelle le directeur général de l'UNESCO, Amadou-Mahtar M'Bow, se vit chargé de procéder à un examen d'ensemble des problèmes de la communication dans la société contemporaine. Le directeur général confia donc cette étude à une commission internationale de seize membres, placée sous la présidence de Sean MacBride, ancien ministre des Affaires étrangères de la République d'Irlande et fondateur d'Amnesty International. Cette commission commença ses travaux en décembre 1977 et tint sa dernière réunion en novembre 1979.

Dans son introduction au rapport publié en $1980^{1}$, Sean MacBride ne cachait pas qu'un certain nombre de désacccords subsistaient sur les moyens d'instaurer ce qu'il était dès lors convenu d'appeler le «Nouvel ordre mondial de l'information et de la communication». Les déséquilibres de l'information mondiale ne pouvaient être supprimés d'un seul coup. Le «Nouvel ordre» était présenté comme un processus plutôt que comme un ensemble de conditions et de pratiques.

La lecture de ce rapport dans les pays occidentaux fut extrêmement critique, d'autant plus que l'UNESCO n'avait pas attendu la fin des travaux de la commission pour adopter le 22 novembre 1978, lors de la vingtième session de sa Conférence générale à Paris, une

1 Voix multiples un seul monde. Communication et société aujourd'hui et demain, Rapport de la Commission internationale d'étude des problèmes de la communication, Paris, UNESCO et Les Nouvelles Editions Africaines, 1980. 
déclaration qui révélait clairement l'orientation prise par l'institution : la «Déclaration sur les principes fondamentaux concernant la contribution des organes d'information au renforcement de la paix et de la compréhension internationale, à la promotion des droits de l'homme et à la lutte contre le racisme, l'apartheid et l'incitation à la guerre». Ces objectifs étaient déclinés en onze articles, dont la formulation ne dissimulait guère les intentions de leurs promoteurs. Ainsi, il appartenait aux organes d'information de «faire entendre la voix des peuples opprimés qui luttent contre le colonialisme, le néo-colonialisme, l'occupation étrangère et toutes formes de discrimination raciale et d'oppression et qui ne peuvent s'exprimer sur leur propre territoire» (article II. 3).

Les critiques occidentaux y lurent un plaidoyer en faveur d'une information au service des pouvoirs locaux, sous le couvert d'une restitution de l'information aux communautés nationales, et surtout une tentative de canaliser la libre circulation des informations et des idées, incompatible avec les principes libéraux de la communication.

La Déclaration de l'UNESCO de 1978 incitait les organisations professionnelles à accorder «une importance particulière» à ses principes dans les codes déontologiques qu'elles établissent. Des rencontres consultatives eurent donc lieu entre des organisations internationales et régionales de journalistes professionnels du monde entier. Lors de la quatrième de ces rencontres, en 1983 à Prague et à Paris, les participants reconnurent la valeur durable de la Déclaration de l'UNESCO de 1978. C'est sur cette base qu'ont été alors élaborés, en dix points, des principes internationaux de l'éthique professionnelle des journalistes, destinés à servir de fondement commun et de source d'inspiration aux codes déontologiques nationaux et régionaux. S'y retrouvent, clairement exprimées par les sept premiers principes, les dispositions partagées par un grand nombre de codes existants : la mission des agents de l'information dans l'intérêt public, la liberté de l'information, l'indépendance et l'intégrité du journaliste, son attachement à la vérité et aux méthodes qui s'y rapportent, le respect de la vie privée et de la dignité de l'homme. La formulation peut en être reconnue comme heureuse, riche et nuancée. En revanche, les trois derniers principes reprennent les grandes orientations de la Déclaration de l'UNESCO constitutive du «Nouvel ordre mondial de 
l'information et de la communication», et apparaissent à ce titre discutables ${ }^{1}$.

La discussion sur le «Nouvel ordre mondial de l'information et de la communication» et sur le rapport MacBride eut des conséquences institutionnelles. L'orientation nouvelle était en contradiction avec l'Acte constitutif de 1'UNESCO qui fait de «la libre circulation des idées par le mot et par l'image» une condition essentielle du rapprochement et de la collaboration entre les nations. L'Union soviétique, ses alliés de l'Europe de l'Est et de nombreux pays de l'hémisphère Sud ne pouvaient guère s'accommoder d'une «libre circulation». Il n'est donc pas étonnant que les États-Unis et le Royaume-Uni aient alors préféré, dans ces conditions, se retirer de l'organisation, respectivement en 1984 et 1985. Ce ne fut pas la seule raison, mais le «NOMIC» y contribua de manière importante. S'ouvrit alors la troisième phase de l'action de l'UNESCO.

Le retour au pouvoir des civils et la fin de la dictature militaire dans plusieurs pays d'Amérique latine dès 1983, la fin des régimes autoritaires dans des pays comme les Philippines et la Corée du Sud, l'instauration de la pereströ̈ka et de la glasnost par Gorbatchev en Union soviétique et enfin la chute du mur de Berlin le 9 novembre 1989, suivie de la dislocation du «bloc» de l'Est, provoquèrent un changement radical, encouragé par le développement même des communications mondiales et des médias électroniques en particulier.

La fin de la guerre froide et de la partition du monde en deux «blocs» provoqua un retour aux principes libéraux de l'information et de la communication. En novembre 1989, la vingt-cinquième Conférence générale de l'UNESCO adopta par consensus une «Nouvelle stratégie de la communication». L'aspect le plus positif des réflexions de la précédente décennie était maintenu, à savoir le renforcement des capacités de communication dans les pays en voie de développement, en vue d'accroître leur participation au processus mondial de la communication. Mais la libre circulation de l'information n'était plus soumise au rééquilibrage de sa diffusion par des dispositions de caractère contraignant. La nouvelle stratégie affirme au contraire que l'objectif est d'assurer «la libre circulation de l'information au niveau international aussi bien que national, ainsi

1 Les principes de l'uNESCO sont couramment présentés comme «Déclaration de l'UNESCO sur les médias», ce qui peut créer un risque de confusion avec la «Déclaration de l'UNESCO» de 1978, fondant le NOMIC. 
qu'une diffusion plus large et mieux équilibrée de l'information, sans aucune entrave à la liberté d'expression».

Cette orientation n'est pas sans conséquence sur la conception même de la déontologie journalistique. Comme le directeur général Frederico Mayor l'a régulièrement rappelé, notamment lors d'un colloque tenu à la Maison de l'UNESCO le 15 mai 1991, «la déontologie de l'information est l'affaire des professionnels et d'eux seuls, et non pas des pouvoirs publics, qu'ils soient nationaux ou internationaux». C'est très exactement revenir sur la position libérale adoptée au début des années cinquante par l'Organisation des Nations Unies elle-même. La même année, la Déclaration de Windhoek confirma cette orientation en réclamant une presse africaine «indépendante et pluraliste».

\section{Les institutions européennes}

Depuis la signature du traité de Rome en 1957, l'Europe communautaire occupe une place prépondérante sur le continent dans les domaines économique et politique. Elle suit le développement des médias et ne manque pas d'émettre, de cas en cas, des recommandations. Le Conseil de l'Europe, qui a été créé en 1949 et dont le siège est à Strasbourg, rassemble un cercle d'États se réclamant de la démocratie, prêts à partager et défendre un patrimoine commun de libertés et de droits individuels : les grands principes consignés dans la Déclaration européenne des droits de l'homme. Le Conseil de l'Europe a été amené à débattre d'un projet ambitieux de régulation des médias.

La Communauté européenne, aujourd'hui l'Union européenne, a toujours fait preuve de réserve en matière d'intervention dans la politique des médias. Elle n'a pu cependant négliger de se préoccuper de la question. La Commission rendit, en date du 14 juin 1984, un important rapport signalant le poids économique et social des médias électroniques: Télévision sans frontières. Livre vert sur l'établissement du marché commun de la radiodiffusion, notamment par satellite et par câble. Ce document fut complété en 1989 par de Nouvelles directives sur les quotas de diffusion d'œuvres européennes, ellesmêmes renforcées par décision du Parlement européen du 14 février 1996. 
Dès le début des années quatre-vingt, le Parlement européen s'est inquiété des dangers que faisaient peser sur le pluralisme des opinions la commercialisation des nouveaux moyens d'information et les monopoles publics ou privés. Au début de la décennie suivante, le Parlement européen adopta une résolution du 16 septembre 1992 sur la concentration des médias et le pluralisme des opinions. Ce texte invitait la Commission et le Conseil à prendre en considération les demandes légitimes des entreprises du secteur des médias imprimés visant à préserver leur base économique, afin de garantir le maintien d'une pluralité de titres. La Commission publia en conséquence, le 23 décembre 1992, un nouveau «Livre Vert» intitulé Pluralisme et concentration des médias dans le marché intérieur. Évaluation de la nécessité d'une action communautaire. Elle y affirme le principe de la «subsidiarité», qui veut que la protection du pluralisme relève en premier lieu des États membres.

Selon la ligne libérale suivie avec constance, les modes d'intervention sont toujours limités et accordés aux objectifs fondamentaux de respect de la liberté d'expression, du pluralisme des informations et des opinions. Les interventions dans le domaine des pratiques et des normes journalistiques sont exceptionnelles. Deux amendements ont été ainsi apportés par le Parlement européen, en décembre 1993, à un important rapport de sa Commission des libertés publiques et des affaires intérieures, portant sur la liberté d'expression du citoyen et la liberté de la presse ou de l'information. Le premier demande que les médias ne diffusent pas d'informations falsifiées ou manipulées. Le second pose que la liberté de recevoir des informations trouve ses limites lorsque 1'honneur, la dignité humaine, la vie privée et la présentation inutile de la violence sont en jeu. Le Parlement européen adopta en outre, le 18 janvier 1994, une résolution visant à assurer la protection du secret des sources d'information des journalistes et invitant les États qui ne l'ont pas encore reconnu à procéder à l'adaptation de leur législation.

La réserve très libérale observée par Bruxelles ne se retrouve pas dans la «grande Europe» de Strasbourg. Le Conseil de l'Europe fait reposer sa politique des médias sur l'article 10 de la Convention européenne des droits de l'homme, signée le 4 novembre 1950 et entrée en vigueur le 3 septembre 1953. Tant la Commission que la Cour européenne des droits de l'homme soulignent de manière constante que la liberté d'expression constitue l'un des fondements essentiels de la 
société démocratique, l'une des conditions primordiales de son progrès et de l'épanouissement de chacun.

Le Conseil de l'Europe n'en a pas moins été tenté de dessiner plus précisément les contours de la liberté et de la responsabilité, de réfléchir aux conditions d'une éventuelle régulation des pratiques des journalistes et des médias, selon une approche qui trouve ses origines dans la doctrine de la «responsabilité sociale», élaborée aux ÉtatsUnis à partir du rapport rendu par la Commission Hutchins en 1947. Cette doctrine tempérait la théorie libérale classique en insistant sur la mise en œuvre de contrepoids internes à l'influence des médias dans la société et de freins visant à empêcher les dérapages.

Une Résolution du 23 janvier 1970 prévoyait déjà que l'organisation interne d'une entreprise médiatique doit garantir la liberté d'expression des rédacteurs responsables, dont l'indépendance devait être préservée. Elle affirmait que pour garantir la responsabilité de la presse, il était souhaitable d'instituer une formation professionnelle des journalistes, d'édicter un code de déontologie et de créer des conseils de la presse. Elle ajoutait que des mesures devaient être prises pour protéger l'individu contre toute ingérence dans l'exercice de son droit au respect de sa vie privée. Dès 1976, l'instance de Strasbourg confia à un Comité directeur sur les moyens de communication de masse (CDMM) la tâche de suivre l'évolution des médias et de rechercher les principes communs propres à assurer leur place dans une société libre et démocratique. La Comité publia en 1980 un premier rapport définissant les «éléments pour une conception européenne des médias». En outre, l'Assemblée parlementaire du Conseil de l'Europe adopta une série de recommandations et de résolutions dans le domaine des médias. Certaines d'entre elles concernent aussi les pratiques journalistiques.

L'initiative la plus marquante a été prise le 1er juillet 1993, lorsque l'Assemblée parlementaire du Conseil de l'Europe adopta une Recommandation et une Résolution relatives à l'éthique du journalisme.

La Recommandation au Comité des Ministres avait notamment pour objet l'étude, en collaboration avec des organisations non gouvernementales telles que la Fédération internationale des journalistes, d'un mécanisme européen d'autocontrôle de l'information, conçu comme un «ombudsman» européen des médias, ainsi que l'adoption d'une déclaration sur l'éthique du journalisme. 
Il revenait à la Résolution de définir les principes de cette éthique journalistique, qui étaient déclinés en trente-huit points. Quelles sont les grandes lignes de ce texte touffu et complexe ?1

Toute réflexion morale sur le journalisme doit partir d'une claire différenciation entre les nouvelles et les opinions, de manière à éviter toute confusion. Les rôles respectifs des éditeurs, des propriétaires de médias et des journalistes sont distingués, ce qui conduit à une rééquilibrage interne : il faut non seulement garantir la liberté des médias dans l'intérêt des citoyens, mais aussi éviter les pressions internes qui mettraient en danger l'indépendance des journalistes. La transparence des entreprises médiatiques doit être assurée en matière de propriété et de gestion. La liberté d'orientation idéologique des éditeurs ou des propriétaires est reconnue, mais elle est limitée «par les exigences incontournables de la véracité des nouvelles et de rectitude morale des opinions, exigées par le droit fondamental des citoyens à l'information». C'est pourquoi les garanties à la liberté d'expression des journalistes qui, «en dernier ressort, transmettent l'information», doivent être renforcées par une clarification de la clause de conscience et par la protection du secret rédactionnel. Des statuts de rédaction doivent être élaborés en vue de réglementer les rapports professionnels des journalistes avec les éditeurs ou propriétaires de médias.

L'information est un droit fondamental du citoyen. Les pouvoirs publics ne peuvent donc considérer qu'ils en sont les propriétaires, ce qui exclut la censure. La même réserve s'applique aux éditeurs ou propriétaires de médias, ainsi qu'aux journalistes eux-mêmes. Les médias n'ont pas à se substituer aux pouvoirs publics ou aux institutions à caractère éducatif ou culturel, telles que l'école. La Résolution refuse de considérer que les médias «représentent l'opinion publique». Ils ne sauraient être érigés en pouvoirs et contre-pouvoirs «sans qu'ils soient représentatifs des citoyens, et assujettis aux contrôles démocratiques des pouvoirs publics». Le journalisme

1 Afin de mettre en évidence les spécificités de cette proposition, il est fait abstraction ici des éléments permanents de la déontologie journalistique, soit : au chapitre de la liberté, l'indépendance des journalistes à l'égard des pouvoirs publics et des milieux économiques, leur droit à un salaire «digne» et à des conditions, des moyens et des instruments de travail «appropriés»; au chapitre de la vérité, le traitement rigoureux de l'information, l'application de méthodes loyales pour l'obtenir, la rectification des informations et des opinions «démontrées fausses ou erronées»; au chapitre du respect de la personne, le droit de réponse, la présomption d'innocence, la protection de la vie privée et de la dignité humaine, le renoncement à l'exploitation inutile de la violence. 
d'investigation est légitime dès lors qu'il est au service de la vérité. Mais il est incompatible avec des campagnes journalistiques engagées sur des prises de position a priori et menées au service d'intérêts particuliers.

$\mathrm{Ni}$ les journalistes ni les éditeurs ou propriétaires ne doivent tirer de profits personnels de l'information. Les premiers en cherchant à acquérir prestige ou influence. Les seconds en considérant l'information comme une marchandise.

La Résolution entre enfin dans le détail de la composition des organes chargés de s'assurer du respect de l'éthique journalistique ainsi définie. Il faut, dit-elle, créer des organismes ou des mécanismes d'autocontrôle, composés d'éditeurs, de journalistes, d'associations de citoyens, de représentants des milieux universitaires et de juges. Les médias s'engageront à rendre publiques les décisions de ces instances. Des recherches critiques sur le travail des médias et des journalistes seront encouragées de la part des organismes eux-mêmes, ainsi que des associations d'utilisateurs des médias et des instituts universitaires spécialisés. L'objectif est d'obtenir «un baromètre de la crédibilité» qui renseignera les citoyens sur la valeur éthique de chaque média comme organisation, voire de tel ou tel journaliste comme individu.

Cette initative fut saluée par la Fédération internationale des journalistes, qui releva toutefois l'ambiguité de certaines formulations. Elle provoqua de vives réactions dans les milieux d'éditeurs. La Fédération internationale des éditeurs de journaux (F.I.E.J.) la considéra comme «l'une des atteintes les plus graves à la liberté et à l'indépendance de la presse de ces dernières années». Elle ne pouvait admettre que l'on cherchât à dicter aux médias leur rôle et leur contenu et les soumettre à un contrôle. Elle s'opposa à toute définition d'un journalisme «honnête» ou «éthique» par des mesures d'encadrement et par la création obligatoire d'un mécanisme international d'«autocontrôle de l'information». La défense des valeurs de la démocratie, ainsi que l'encouragement de la compréhension mutuelle, de la tolérance et de la confiance relèvent, dit-elle, de la libre appréciation de chacun. En imposer l'obligation restreint la liberté d'expression. Enfin, la F.I.E.J. ne pouvait accepter les limites imposées aux éditeurs quant à leur liberté de choisir l'orientation de leur publication, quant à leur droit de chercher à exercer une influence sur l'opinion et quant à leur action en vue d'assurer l'assise commerciale de leur entreprise. 
Cette opposition entraîna le renvoi de la Recommandation au Comité directeur sur les moyens de communication de masse. Celui-ci organisa le 9 novembre 1993 une audition des milieux intéressés sur le thème «Libertés journalistiques et droits de l'homme». Il rendit des conclusions très réservées, craignant qu'une réglementation sur l'éthique du journalisme ne servît de prétexte à une ingérence des pouvoirs publics dans la liberté des médias. De la même manière, le CDMM exprima son scepticisme sur l'opportunité d'installer un «ombudsman» européen. Dans le même temps, la section Médias de la Direction des droits de l'homme du Conseil de l'Europe élaborait un avant-projet de principes sur le journalisme et les droits de l'homme.

Le Comité des Ministres du Conseil de l'Europe décida finalement de ne pas suivre l'Assemblée parlementaire, dans une réponse adoptée le 21 mars 1994. Il reconnut l'importance de médias indépendants et pluralistes pour la libre circulation des informations et des idées, le droit des individus et de la société en général à les exprimer, communiquer et recevoir librement, et par conséquent le devoir pour les médias d'exercer leurs activités de manière responsable. Mais il fut d'avis que le recours à une intervention législative n'était pas le moyen le plus approprié de concilier la liberté des médias avec d'autres droits et d'autres valeurs. Il exprima la crainte que l'orientation adoptée par l'Assemblée parlementaire n'incitât les pouvoirs politiques à porter atteinte à la liberté des médias sous prétexte de promouvoir un journalisme responsable. Il s'opposât formellement à l'idée d'un «ombudsman européen des médias», qui aurait pour tâche de vérifier l'exactitude des informations. Une telle institution serait en contradiction avec le rôle du Conseil de l'Europe comme garant de la liberté de la presse. Il ne jugea pas non plus nécessaire d'adopter une déclaration sur l'éthique du journalisme, voyant bien la difficulté qu'il y aurait à définir, en vue de la promouvoir, une présentation impartiale des informations, en raison même de l'existence de lignes éditoriales diverses, constitutives du pluralisme des médias. Le Comité des Ministres soutint en revanche toute mesure visant à encourager un usage critique des médias par le public, l'éducation aux médias dans les programmes scolaires et la constitution d'associations d'usagers indépendantes, aspects peu discutés de la Recommandation.

Le débat trouva sa conclusion lors de la quatrième Conférence ministérielle européenne sur la politique des communications de masse, qui se tint à Prague les 7 et 8 décembre 1994. La conférence 
adopta une résolution fondée sur un rapport présenté par la délégation de l'Autriche, sous le titre Les media dans une société démocratique. Elle ramena le Conseil de l'Europe à l'équilibre libéral entre le droit à l'information et à l'expression des citoyens et le droit de chacun à la protection de sa vie privée et de sa personne ${ }^{1}$.

\section{Un débat récurrent}

Les tentatives des organisations internationales, constituées par des États, d'orienter l'éthique journalistique ont donc toutes échoué. La responsabilité d'en définir les termes a été à chaque fois renvoyée aux organisations professionnelles elles-mêmes. Le débat ne s'en trouve pas clos pour autant. Pour deux raisons.

La première est que la volonté de réguler par le haut (instruments internationaux, organes contrôlés par les pouvoirs publics) anime et animera d'autres initiatives. En Suisse, une proposition parlementaire préconise l'installation d'un médiateur pour la presse écrite (sur le modèle de l'autorité indépendante fonctionnant déjà pour les médias électroniques), alors qu'existe depuis 1977 un Conseil de la presse, instance d'autorégulation qui fut mise en place par la Fédération suisse des journalistes, la plus importante organisation professionnelle du pays, et qui veille au respect des règles déontologiques. La discussion sur la proposition parlementaire est en cours; elle semble à ce jour s'orienter vers un respect du principe de l'autorégulation. Au mois de septembre 1998, l'Association mondiale des conseils de la presse («World Association of Press Councils») s'est réunie à Istanbul. Cet aréopage est très peu représentatif : il ne regroupe que seize conseils, professionnels ou publics, dont un très petit nombre appartient à des pays de tradition libérale. Lors d'un colloque, il débattit de l'opportunité d'élaborer un code de conduite international. Faut-il donc s'attendre à un retour au «nouvel ordre mondial de l'information»? Le risque est ténu, mais ce serait s'avancer que de le considérer comme écarté à jamais: sous le couvert de «l'équilibre» ou

1 Une synthèse de cette discussion a été opérée par Pierre-Ami CHEvaliER, «La presse suisse et l'Europe», dans un ouvrage publié par l'Union romande des éditeurs de journaux et périodiques (U.R.J.) à l'occasion de son 75e anniversaire, Presse romande : du miracle à la réalité, 1996. 
de «l'objectivité» de l'information se cache souvent l'intention des pouvoirs publics d'en contrôler le cours' .

La seconde raison à la permanence des entreprises de régulation autoritaires tient au comportement des médias eux-mêmes, aux défaillances individuelles et plus encore au laisser-faire de l'ensemble du système médiatique, qui ne semble se préoccuper de ses écarts ou ses dérives que sous la menace de mesures légales, ainsi que l'illustre depuis une dizaine d'années le débat en Grande-Bretagne. La tâche des défenseurs du libéralisme serait plus aisée, et sans doute plus convaincante pour le public, si l'assurance était donnée par les professionnels, des journalistes comme individus aux entreprises médiatiques comme organisations, d'en respecter pleinement les orientations. Le libéralisme, en matière d'information, ne tient pas tout entier dans la liberté d'assurer le commerce des nouvelles et de donner en spectacle l'actualité du monde.

1 Voir l'article de Daniel VeRNET, «Le retour du "nouvel ordre mondial de l'information" ?», Le Monde, 24 septembre 1998. 